\section{THE STRUCTURAL CHANGE OF HEME OXYGENASE UPON HEME BINDING}

M Sugishima $^{1} \mathrm{H} \mathrm{Sakamoto}^{2}$ Y Kakuta ${ }^{3}$ Y Omata $^{2}$ S Hayashi $^{2}$ M Noguchi $^{2} \mathrm{~K}$ Fukuyama

${ }^{1}$ Graduate School of Science, Osaka University Department of Biology 1-1 Machikaneyama-Cho TOYONAKA 560-0043 JAPAN ${ }^{2}$ Department of Medical Biochemistry, Kurume University School of Medicine, 67 Asahi-machi, Kurume 830-0011, Japan ${ }^{3}$ Department of Bioscience and Biotechnology, Graduate School of Bioresource and Bioenvironmental Sciences, Kyushu University, 6-10-1, Hakozaki, Fukuoka 812-8581, Japan

The incorporation of heme into apo-forms of hemoproteins is an indispensable process for them to acquire biological functions. Heme oxygenase (HO) catalyzes the oxidative cleavage of protoheme to biliverdin utilizing dioxygens and reducing equivalents from cytochrome $\mathrm{P} 450$ reductase. HO is not a hemoprotein by nature, but once it binds heme, it behaves like a hemoprotein. Because apo-states of most hemeproteins are not native forms and unstable, HO (apoHO) could be a useful model to investigate the formation of holohemoproteins from their apo-states. Rat apoHO was crystallized in hemihedral twin with three molecules in an asymmetric unit and its crystal structure was determined at $2.55 \AA$ Å resolution.

Although the structure of apoHO is similar to the structure of its complex with heme (HO-heme), the structure around the hemepocket shows distinct differences. In apoHO, the proximal helix (A-helix) is disordered and the following helix (B-helix), a portion of which builds the hemepocket, is shifted toward the hemepocket. Notably, Gln38 in the B-helix is shifted toward a position where the heme is present in HO-heme. In HO-heme, Gln38 is hydrogen-bonded to Glu29 that is located at the C-terminal side of the A-helix in $\mathrm{HO}$-heme. This finding suggests that this hydrogen bond restrains the angle between the A- and B-helices in HO-heme. In addition, the secondary structure of distal helix in apoHO changes to random coil. These structural features of apoHO imply that the orientation of the proximal helix and the position of His25 are fixed upon heme binding.

Keywords: HEME PROTEIN BINDING TWINNING

\section{Acta Cryst. (2002). A58 (Supplement), C120 \\ CRYSTAL STRUCTURE OF CHLOROPLASTIC ASCORBATE PEROXIDASE FROM TOBACCO PLANTS}

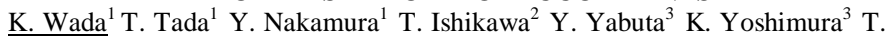
Takeda $^{3}$ S. Shigeoka ${ }^{3}$ K. Nishimura ${ }^{1}$

${ }^{1}$ Osaka Prefecture University Research Institute for Advanced Science and Technology Gakuen-Cho 1-2 SAKAI OSAKA 599-8570 JAPAN ${ }^{2}$ Faculty of Life and Environmental Science, Shimane University, Shimane 690-8504, Japan ${ }^{3}$ Department of Food and Nutrition, Faculty of Agriculture, Kinki University, Nara 631-8505, Japan

Ascorbate peroxidase (APX) isoenzymes are known to play an important role in scavenging active oxygen species in higher plants. One of the characteristics of APX isoenzymes is their instability in ascorbate-depleted medium. Especially, the chloroplastic APX loses its activity within several minutes, when the concentration of ascorbate is below 20 microM. In contrast, the activity of cytosolic APX (cAPX) is retained at least for several hours even in an ascorbate-depleted medium. We analyzed the crystal structure of stromal APX (sAPX), which is a chloroplastic isoenzyme, to obtain some information about the mechanisms for its rapid inactivation. The sAPX from tobacco plants was overexpressed in Escherichia coli and purified by chromatography. The enzyme was crystallized, and data were collected using synchrotron radiation at KEK-PF. The structure was solved and refined to an R-factor of $19.8 \%$. The overall structure of the sAPX was similar to that of cAPX with a substantial difference in a random-coil structure located in the vicinity of heme. The heme seemed to be anchored in place by similar sets of interactions but with an interesting variation. His169 of cAPX forms H-bonds with the propionate side chain of heme. The side chain of Arg169 in SAPX, corresponding to His169 of cAPX, was in the opposite direction from the heme, forming two H-bonds with carbonyl groups in the random-coil structure. These findings suggested that the rapid inactivation of sAPX is due to the characteristic conformation of Arg169.

Keywords: ASCORBATE PEROXIDASE TOBACCO PLANTS INSTABILITY
Acta Cryst. (2002). A58 (Supplement), C120

CRYSTAL STRUCTURE OF WATER-SOLUBLE CHLOROPHYLL PROTEIN FROM LEPIDIUM VIRGINICUM

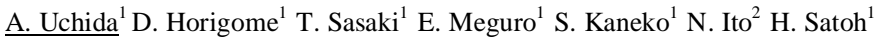
I. Oonishi ${ }^{1}$

${ }^{1}$ Toho University Biomolecular Science Miyama 2-2-1, FUNABASHI $274-$ 8510 JAPAN

${ }^{2}$ Department of Chemistry, Faculty of Science, Toho University

Generally, chlorophylls (Chls) in higher plants are associated with membrane proteins. However, a water-soluble chlorophyll protein (WSCP) was found in Chenopodium album in 1963 [1]. WSCPs have been also detected in the Brassicaceae, Chenopodiaceae, Polygonaceae and Amaranthaceae plants [2]. The physiological functions of WSCPs have not been known so far, but it has been suggested that WSCPs are related to drought-induced proteins. As Chl $\mathrm{a} / \mathrm{b}$ ratios in WSCPs differ from the ratio in total leaf extract, WSCPs may discriminate between $\mathrm{Chl} a$ and $\mathrm{b}$.

The molecule of WSCP from Lepidium virginicum consists of four subunits with a molecular mass of $19 \mathrm{kDa}$. Crystals of the WSCP belong to space group $P 22_{1} 2_{1}$ with unit-cell dimensions of $\mathrm{a}=83.1, \mathrm{~b}=122.4, \mathrm{c}=73.3 \AA$. The threedimensional structure was solved using the MIR method. The molecule contains four Chls. Two Chls form a sandwich structure in the molecular core and two sandwich structures are approximately orthogonal to each other. Four phytyl groups of Chls have folded conformations and locate in the space surrounded by four $\mathrm{Chl}$ planes. The carbonyl oxygen atom of Pro 32 binds to the $\mathrm{Mg}$ atom of Chls. The distance between $\mathrm{Mg}$ atoms in the sandwich structure is about $10 \AA$.

References

[1] Yakushiji, E., Uchino, K., Sugimura, Y., Shiratori, I. and Takamiya, F. (1963) Biochim. Biophys. Acta 75, 293-298

[2] Takamiya, A. (1973) Carnegie Inst. Wash. Yearbook 72, 330-336

Keywords: CHLOROPHYLL LEPIDIUM WSCP

Acta Cryst. (2002). A58 (Supplement), C120

CRYSTAL STRUCTURES OF N-CARBAMOYL-D-AMINO-ACID AMIDOHYDROLASE-SUBSTRATE COMPLEXES: STRUCTURAL INSIGHTS INTO THE BINDING POCKET

W. Wang C. Chen J. Liu

National Tsing Hua University Department of Life Sciences 101 Sec. 2 KungFu Rd. HSINCHU 300 TAIWAN

The crystal structure of N-carbamoyl-D-amino-acid amidohydrolase (DNCAase) from Agrobacterium radiobacter, an industrial biocatalyst for the production of valuable D-amino acids, has determined to a resolution of 1.95 angstrom. The substitution of Cys172 with Ser or Ala, a residue implicated in a nucleophilic attack of the $\mathrm{C}$ atom of the carbamoyl group in a substrate, inactivated the enzyme but did not lose the ability to bind substrate. The mutation of Arg175 or Arg176 with Ala, respectively, abolished both binding and enzymatic activities. Crystal structures of Cys172Ala and Cys172Ser were determined respectively in its native form and in complex form with $\mathrm{N}$ carbamoyl-D-p-hydroxyphenylglycine.

Analysis of these structures suggest that (i) the overall protein folding, side chain conformations and active sites of both mutants, mutant-substrate complexes are isomorphous with those of wild-type enzyme, (ii) the mutated side chains are pointed for catalysis in a similar conformation as that observed for the wild-type D-NCAase, and (iii) within the identified substrate binding sites, a number of residues including Arg175 and Arg176 are involved in interacting with the substrate.

Keywords: AMINO ACID AMIDOHYDROLASE CYS172, D-NCAASE, BINDING 